ACCepted for publication in the Astrophysical Journal (Letters) 2010 April 9

Preprint typeset using LATEX style emulateapj v. 11/10/09

\title{
EVIDENCE FOR DELAYED MASSIVE STAR FORMATION IN THE M17 PROTO-OB ASSOCIATION
}

\author{
Matthew S. Povich ${ }^{1}$ \\ Department of Astronomy and Astrophysics, The Pennsylvania State University, 525 Davey Laboratory, University Park, PA 16802; \\ povich@astro.psu.edu \\ AND \\ BARBARA A. WHITNEY \\ Space Science Institute, 3100 Marine Street, Suite A353, Boulder, CO 80303; bwhitney@spacescience.org \\ Accepted for publication in the Astrophysical Journal (Letters) 2010 April 9
}

\begin{abstract}
Through analysis of archival images and photometry from the Spitzer GLIMPSE and MIPSGAL surveys combined with 2MASS and MSX data, we have identified 488 candidate young stellar objects (YSOs) in the giant molecular cloud M17 SWex, which extends 50 pc southwest from the prominent Galactic H II region M17. Our sample includes $>200$ YSOs with masses $>3 \mathrm{M}_{\odot}$ that will become B-type stars on the main sequence. Extrapolating over the stellar initial mass function (IMF), we find that M17 SWex contains $>1.3 \times 10^{4}$ young stars, representing a proto-OB association. The YSO mass function is significantly steeper than the Salpeter IMF, and early O stars are conspicuously absent from M17 SWex. Assuming M17 SWex will form an OB association with a Salpeter IMF, these results reveal the combined effects of (1) more rapid circumstellar disk evolution in more massive YSOs and (2) delayed onset of massive star formation.
\end{abstract}

Subject headings: circumstellar matter — ISM: clouds — stars: formation — stars: luminosity function, mass function

\section{INTRODUCTION}

Elmegreen \& Lada (1976) discovered an extended molecular cloud associated with the well-known Galactic $\mathrm{H}$ II region M17. The size $(70 \mathrm{pc} \times 15 \mathrm{pc}$ at $d=2.1 \mathrm{kpc})$, mass $\left(>2 \times 10^{5} \mathrm{M}_{\odot} ;\right.$ Elmegreen et al. 1979), and fragmentary morphology of this cloud were reminiscent of the largest Galactic OB associations. Elmegreen \& Lada (1976) suggested that an extended OB association will eventually form near M17, and they noted an apparent progression of ages among the known OB populations, H II regions, and molecular cloud cores across the complex. The M17 complex became an observational touchstone for the theory of sequential triggered massive star formation by propagating ionization fronts (Elmegreen \& Lada 1977).

While the M17 H II region itself and the interface between the ionization front and the massive molecular core known as M17 SW have remained the focus of intense study (Chini \& Hoffmeister 2008, and references therein), interest in the extended molecular cloud, which we name M17 SWex (since M17 SW is ambiguous), has gradually waned. M17 SWex lacks very massive stars; a handful of low-luminosity compact and ultracompact H II regions have been identified, but they are insufficiently powerful to trigger massive star formation throughout the cloud (Elmegreen et al. 1979; Jaffe \& Fazio 1982).

M17 SWex presents one of the most striking infrared dark cloud (IRDC) morphologies in the Galaxy, revealed in great detail by the Spitzer Space Telescope as part of the Galactic Legacy Infrared Mid-Plane Survey Extraordinaire (GLIMPSE; Beniamin et al. 2003) and the Multiband Imaging Photometer for Spitzer Galactic Plane Sur-

\footnotetext{
${ }^{1}$ NSF Astronomy and Astrophysics Postdoctoral Fellow
}

vey (MIPSGAL; Carev et al. 2009). IRDCs have been the subject of much recent work because they are laboratories for studying the initial conditions for massive (OB stars, $m \geq 8 \mathrm{M}_{\odot}$ ) star formation (Rathborne et al. 2006; Beuther \& Sridharan 2007; Butler \& Tan 2009).

In this Letter, we use archival GLIMPSE and MIPSGAL data to find and characterize individual young stellar objects (YSOs) throughout the $\sim 1^{\circ}$ extent of M17 SWex. We model the mass function of the YSO population and find that it bears the imprints of both massdependent circumstellar disk evolution and delayed massive star formation in M17 SWex.

\section{DATA ANALYSIS AND MODELING}

\subsection{Selection of Candidate YSOs By SED Fitting of GLIMPSE Catalog Sources}

The infrared (IR) spectral energy distributions (SEDs) of YSOs are dominated by emission from dusty circumstellar disks and infalling envelopes. For details on our procedure for identifying candidate YSOs we refer the reader to Povich et al. (2009, hereafter P09) and Smith et al. (2010). Here we summarize the main analysis steps. Using a $\chi^{2}$-minimization SED fitting tool (Robitaille et al. 2007), we fit reddened Kurucz (1993) stellar atmospheres (varying $A_{V}$ from 0 to $40 \mathrm{mag}$ ) to the SEDs of 64,820 sources in the highly-reliable GLIMPSE Point Source Catalog located within a $1^{\circ} \times 0.75$ field encompassing M17 SWex. Only sources detected in $N_{\text {data }} \geq 4$ of the 7 Catalog bands $(\lambda=1-8 \mu \mathrm{m})$ were fit. The best-fit stellar atmosphere model was a poor fit $\left(\chi_{\min }^{2} / N_{\text {data }}>4 ; \chi_{\min }^{2}\right.$ is the goodness-of-fit parameter $)$ for 1498 sources; hence they are possible YSOs.

We then filtered out sources with IR excess emission appearing in only the IRAC [5.8] or [8.0] bands using the Smith et al. (2010) color criteria, modified to de- 
redden the [3.6] - [4.5] colors of background stars viewed through the M17 SWex cloud (using the extinction law of Indebetouw et al. 2005). This step was necessary to distinguish intrinsically red objects from sources affected by systematic photometric errors; IRAC [5.8] and [8.0] are less sensitive and more affected by nebular emission compared to IRAC [3.6] and [4.5]. We discarded 592 sources, leaving 906 candidate YSOs in our initial sample.

\subsection{Constraints on SED Models from MIPS $24 \mu \mathrm{m}$ Aperture Photometry}

We fit YSO model SEDs from Robitaille et al. (2006, hereafter RW06) to the available IR photometry of each candidate YSO in the initial sample to constrain luminosity, mass, and evolutionary stage (Stage 0/I, dominated by infalling envelope; Stage II, optically thick circumstellar disk; Stage III, optically thin disk; or Ambiguous; see P09). Photometry at $\lambda>10 \mu \mathrm{m}$ is required to constrain bolometric luminosities and often to distinguish Stage 0/I from Stage II sources (RW06; Indebetouw et al. 2007). We located the position of each YSO candidate in a MIPSGAL $24 \mu \mathrm{m}$ enhanced mosaic (Carey et al. 2009) and extracted fluxes using aperture photometry. We compared the aperture photometry results to fluxes extracted via PSF fitting with the GLIMPSE pipeline for a subset of sources and found excellent agreement for aperture radius $3.5^{\prime \prime}$ with background annulus $7^{\prime \prime}$ to $13^{\prime \prime}$. This choice of aperture re-

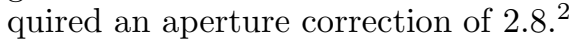

Aperture photometry detected more $24 \mu \mathrm{m}$ sources than PSF fitting. For the $35 \%$ of sources lacking $24 \mu \mathrm{m}$ detections we placed $5 \sigma$ upper limits, a useful constraint for the SED fitting. The brightest $\sim 1 \%$ of sources were saturated in the MIPSGAL images; for these we treated the extracted fluxes as lower limits. For the $24 \mu \mathrm{m}$ detections we set the minimum uncertainty to $15 \%$ to avoid overly weighting the $24 \mu \mathrm{m}$ flux in the model fits in case of residual systematic uncertainties. The results of our aperture photometry are presented in Table 1. One potential source of systematics is the mid-IR extinction law for $\lambda>10 \mu \mathrm{m}$. Recent work has found similar reddening at both $24 \mu \mathrm{m}$ and $8 \mu \mathrm{m}$ in dense molecular clouds (e.g. Flaherty et al. 2007). We used the high-density $\left(A_{K} \geq 1\right)$ mid-IR extinction law of McClure (2009) when fitting the RW06 models to the SEDs of our initial YSO sample.

\subsection{MSX Detections of Luminous Candidate YSOs}

We incorporate $8-21 \mu \mathrm{m}$ photometry from the $M S X$ Galactic Plane Survey Point Source Catalog (Price et al. 2001) into our YSO sample in 2 ways: (1) Spatial correlation of $M S X$ sources with the initial YSO sample and (2) visual identification of bright sources located near the IRDCs in the GLIMPSE and MIPSGAL mosaics that were excluded from the the GLIMPSE Cata$\log$ due to saturation. Moderately saturated sources were included in the more complete GLIMPSE Point Source Archive, and we added $15 M S X$ sources with Archive matches to the initial YSO sample. The $\sim 3 \%$ of the YSO sample with $M S X$ counterparts (indicated in Table 11), include the most luminous YSOs in the cloud.

2 MIPS Instrument Handbook v1.0, http://ssc.spitzer.caltech.edu/mips/mipsinstrumenthandbook/
The $M S X$ data provide strong constraints on the mid-IR SEDs along with replacements for Spitzer measurements suffering from saturation at 8.0 or $24 \mu \mathrm{m}$.

\subsection{Removal of Contaminants from the YSO Sample}

In addition to YSOs, the initial sample may contain the following classes of contaminants: variable stars, dusty asymptotic giant branch (AGB) stars, unresolved planetary nebulae, and background galaxies. The SEDs of variable stars and many AGB stars cannot be fit by stellar atmospheres or YSO models; $15 \%$ of the initial sample were discarded for this reason. Of the remaining contaminants, luminous AGB stars are the most important, as they can masquerade as massive YSOs (P09). The majority of AGB stars have [8.0] - [24] $<2.2 \mathrm{mag}$ (Whitney et al. 2008), and applying this color cut removes an additional $5 \%$ of sources.

While we expect YSOs associated with M17 SWex to be clustered, contaminating sources, including foreground YSOs, will be distributed uniformly. Following P09, we used a cluster-finding algorithm to select sources exhibiting a significant degree of clustering with respect to a "control" region located away from the molecular cloud. The control field sampled was a $0.38 \times 0.35$ box centered at $(l, b)=(13.89,-0.76)$ with source density $270 \mathrm{deg}^{-2}$, yielding a similar average source separation $\left(\Theta_{\text {con }}=3.84^{\prime}\right)$ to the P09 control field. The surface density ratio between the control field and the entire field is $\Sigma_{\text {con }} / \Sigma_{\text {targ }}=0.27$, which shows that M17 SWex presents a significantly larger overdensity of candidate YSOs versus contaminants compared to the M17 molecular cloud sample analyzed by P09. We thereby split the initial sample into a clustered population $(\sim 65 \%)$ comprised of YSOs associated with M17 SWex and a distributed component ( $\sim 35 \%$ ) dominated by unassociated contaminants.

\section{RESULTS}

\subsection{Final YSO Sample}

The clustered population of 488 sources comprises our final YSO sample. The YSOs exhibit a highly structured spatial distribution, strongly clustered along the IRDC filaments with numerous sub-clusters (Fig. 11). Using the set $i$ of well-fit models for each YSO (defined by $\left.\chi_{i}^{2}-\chi_{\min }^{2} \leq 2 N_{\text {data }}\right)$, we construct probability distributions of bolometric luminosity $L_{\mathrm{bol}}$, mass of central star $M_{\star}$, and evolutionary stage (see P09 for details). We allowed the model fitting tool to accept a range of distances from 1.9 to $2.3 \mathrm{kpc}$, hence incorporating the uncertain distance to the M17 complex into the uncertainties on the resultant model parameters (P09). The results for each source are summarized in Table 11. The final sample includes 133 Stage 0/I, 276 Stage II, 4 Stage III, and 75 YSOs with ambiguous stage. The paucity of Stage III objects is a selection effect and suggests that virtually all of the ambiguous sources are ambiguous between Stages 0/I and II. Stage 0/I objects are more tightly clustered than are Stage II, supporting the idea that the stages represent an evolutionary sequence. The high fraction of Stage $0 /$ I sources $\left(N_{\text {OI }} / N_{\text {II }} \approx 0.5\right)$ confirms the extreme youth of the population.

Among the final sample, 68 YSOs exhibit excess $4.5 \mu \mathrm{m}$ emission over the best-fit YSO model. These may be un- 


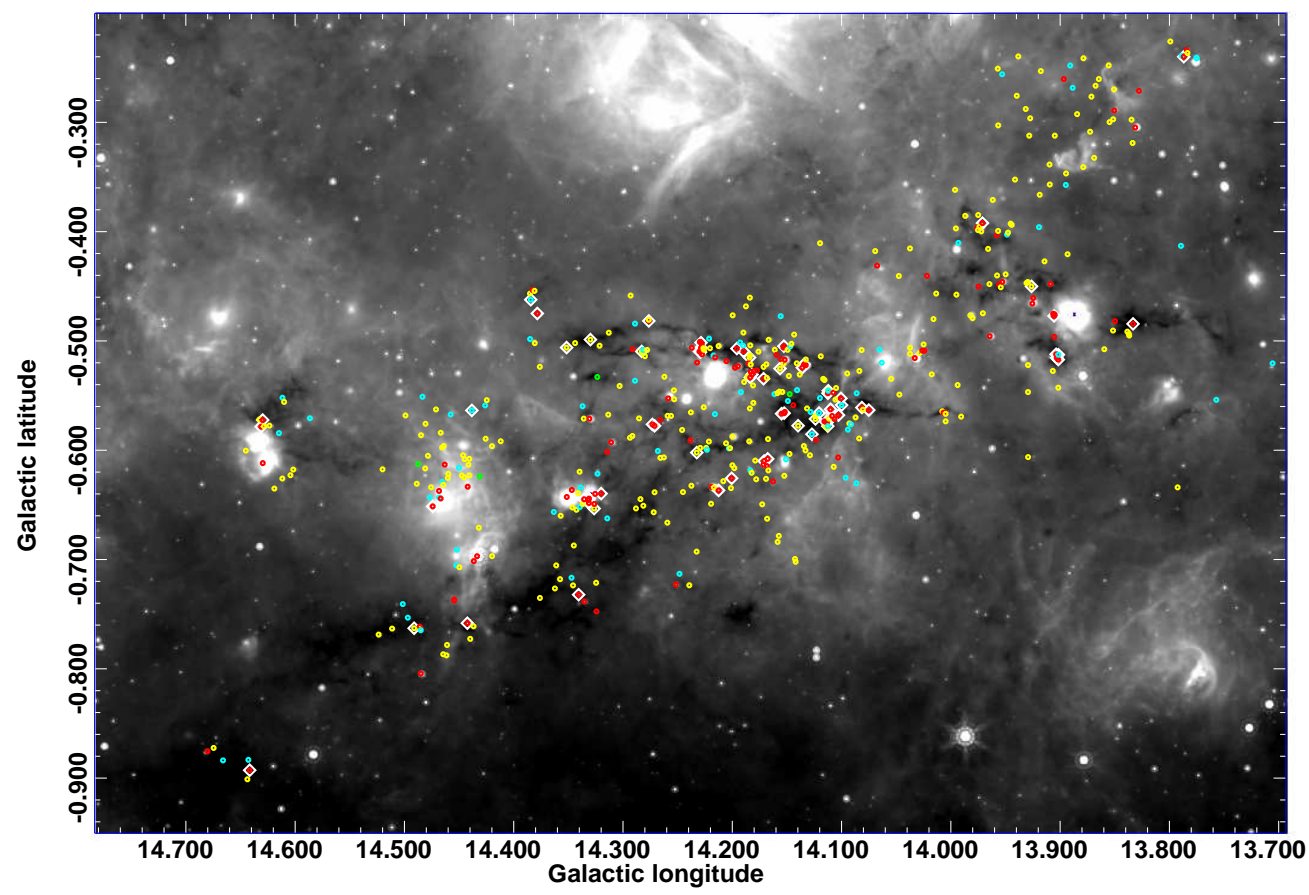

FIG. 1. - MIPSGAL $24 \mu \mathrm{m}$ mosaic image with positions of the 488 YSOs in the final sample overlaid. Colors identify YSO evolutionary stages: $r e d=$ Stage 0/I, yellow=Stage II, green=Stage III, cyan=ambiguous. YSOs with $4.5 \mu \mathrm{m}$ excess emission are highlighted with white diamonds.

TABLE 1

Basic Properties of Candidate YSOs

\begin{tabular}{|c|c|c|c|c|c|c|c|c|c|c|c|c|}
\hline $\begin{array}{l}\text { Cat. } \\
\text { No. }\end{array}$ & $\begin{array}{l}\text { GLMA } \\
\text { Name }\end{array}$ & $\begin{array}{l}{[24]^{\mathrm{a}}} \\
(\mathrm{mag})\end{array}$ & $q_{24}{ }^{\mathrm{b}}$ & $\begin{array}{c}\left\langle\log L_{\mathrm{bol}}\right\rangle \\
\left(\mathrm{L}_{\odot}\right)\end{array}$ & $\begin{array}{c}\sigma\left(\log L_{\mathrm{bol}}\right) \\
\left(\mathrm{L}_{\odot}\right)\end{array}$ & $\begin{array}{l}\left\langle M_{\star}\right\rangle \\
\left(\mathrm{M}_{\odot}\right)\end{array}$ & $\begin{array}{c}\sigma\left(M_{\star}\right) \\
\left(\mathrm{M}_{\odot}\right)\end{array}$ & $\begin{array}{l}\left\langle\log \dot{M}_{\text {env }}\right\rangle^{\mathrm{c}} \\
\left(\mathrm{M}_{\odot} \mathrm{yr}^{-1}\right)\end{array}$ & $\begin{array}{c}\sigma\left(\log \dot{M}_{\text {env }}\right) \\
\left(\mathrm{M}_{\odot} \mathrm{yr}^{-1}\right)\end{array}$ & Stage & $M S X$ ? & $\begin{array}{l}4.5 \mu \mathrm{m} \\
\text { Excess? }\end{array}$ \\
\hline 200 & G014.1482-00.5321 & 7.7 & 1 & 0.7 & 0.3 & 1.3 & 0.6 & -5.9 & 2.6 & II & & \\
\hline 201 & G014.1491-00.5550 & 6.1 & 1 & 1.5 & 0.2 & 2.6 & 0.9 & -5.2 & 2.4 & A & & \\
\hline 202 & G014.1494-00.5081 & 6.4 & 3 & 2.1 & 1.2 & 3.5 & 0.9 & -5.7 & 2.4 & II & & \\
\hline 203 & G014.1508-00.6084 & 3.3 & 1 & 2.7 & 0.7 & 3.9 & 2.5 & -4.2 & 1.7 & A & & \\
\hline 204 & G014.1519-00.5650 & 6.0 & 1 & 1.1 & 0.8 & 1.1 & 1.0 & -4.7 & 2.1 & $0 / \mathrm{I}$ & & \\
\hline 205 & G014.1520-00.5173 & 0.6 & 2 & 3.6 & 0.4 & 8.4 & 1.5 & -3.2 & 1.5 & $0 / \mathrm{I}$ & $\checkmark$ & \\
\hline 206 & G014.1524-00.6234 & 6.1 & 1 & 1.8 & 0.2 & 2.8 & 0.3 & -7.2 & 2.9 & II & & \\
\hline 207 & G014.1525-00.5661 & 6.1 & 1 & 1.7 & 1.1 & 1.2 & 0.9 & -3.7 & 0.6 & $0 / \mathrm{I}$ & & $\checkmark$ \\
\hline 208 & G014.1528-00.5048 & 5.8 & 1 & 1.5 & 0.9 & 1.6 & 1.5 & -4.0 & 1.8 & $0 / \mathrm{I}$ & & $\checkmark$ \\
\hline 209 & G014.1530-00.5209 & 5.3 & 1 & 1.8 & 0.2 & 2.8 & 0.6 & -5.5 & 2.4 & II & & \\
\hline 210 & G014.1532-00.5061 & 6.1 & 3 & 1.5 & 0.9 & 2.3 & 0.4 & -4.0 & 0.1 & $0 / \mathrm{I}$ & & \\
\hline 211 & G014.1542-00.5467 & 7.3 & 1 & 1.1 & 0.7 & 1.9 & 1.0 & -5.9 & 2.6 & II & & \\
\hline 212 & G014.1549-00.5666 & 4.7 & 1 & 1.5 & 0.9 & 1.0 & 1.1 & -4.3 & 1.8 & $0 / \mathrm{I}$ & & $\checkmark$ \\
\hline 213 & G014.1552-00.5169 & 3.6 & 1 & 1.9 & 1.1 & 2.8 & 1.4 & -4.1 & 1.9 & $0 / \mathrm{I}$ & & \\
\hline 214 & G014.1557-00.4774 & 5.8 & 1 & 1.6 & 1.0 & 2.2 & 1.1 & -4.8 & 2.1 & $\mathrm{~A}$ & & \\
\hline
\end{tabular}

Note. - Table 1 is available in its entirety in the electronic edition of the Astrophysical Journal Letters. A portion is reproduced here for guidance regarding its form and content.

a Typical uncertainty on $[24]$ is $\sim 0.1$ mag.

b $24 \mu \mathrm{m}$ quality flag: $0=$ non-extraction, $1=$ detection, $2=$ lower limit, $3=$ upper limit.

${ }^{c}$ If $\left\langle\dot{M}_{\text {env }}\right\rangle<10^{-9} \mathrm{M}_{\odot} \mathrm{yr}^{-1}$, accretion has effectively ceased, hence $\left\langle\log \dot{M}_{\text {env }}\right\rangle$ is undefined.

resolved analogs of the "extended green objects" (EGOs), candidate accretion-powered young massive stellar outflows (Cyganowski et al. 2008). The RW06 models do not include outflows, which can produce strong molecular line emission in the $4.5 \mu \mathrm{m}$ band. We set the $4.5 \mu \mathrm{m}$ fluxes to upper limits for the SED fitting in these cases. The majority of $4.5 \mu \mathrm{m}$ excess sources are associated with tight clusters of Stage 0/I YSOs (Fig. 1 and Table 1). Two such groups coincide with known EGOs (G014.330.64 and G014.63-0.58; Cyganowski et al. 2008).

\subsection{Observed YSO Mass Function}

Given the GLIMPSE sensitivity limits and the $2.1 \mathrm{kpc}$ distance to M17, our YSO sample is nearly complete for $m=M_{\star} \gtrsim 3 \mathrm{M}_{\odot}$, corresponding to main-sequence spectral types earlier than A0 (P09). For this mass range, the pre-main-sequence evolutionary tracks are horizontal (Bernasconi \& Maeder 1996; Siess et al. 2000), and YSOs follow the zero-age main sequence mass-luminosity relation (Kang et al. 2009).

Summing the probability distributions of $M_{\star}$ from the 


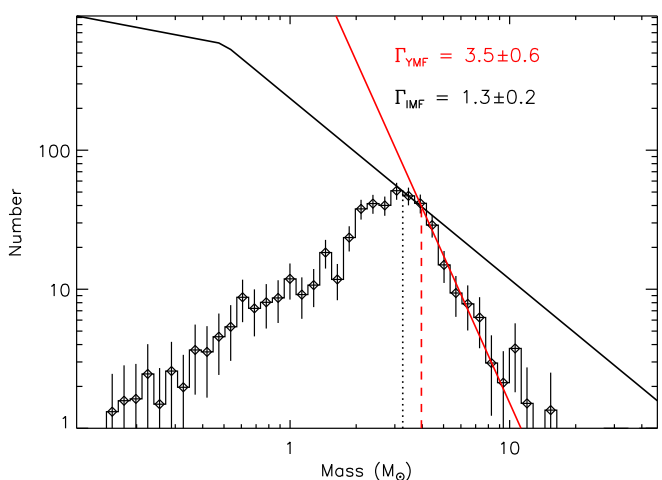

FIG. 2.- YMF plot (histogram points with error bars). Two IMFs are overplotted: power-law fit to the high-mass tail of the YMF $\left(m \gtrsim 4 \mathrm{M}_{\odot} ;\right.$ red $)$ and Kroupa (2001) IMF scaled to match the YMF peak at $\sim 3.2 \mathrm{M}_{\odot}$ (dotted line). The IMFs intersect at $M_{c}=3.9 \mathrm{M}_{\odot}$ (dashed line).

model fits to each YSO, we construct the YSO mass function (YMF; P09) for the 488 sources in our final sample (Fig. 2). The YMF has the same form as the stellar initial mass function (IMF),

$$
\Phi(\log m)=d N / d \log m \propto m^{-\Gamma},
$$

where $d N$ is the number of stars in the (logarithmic) mass interval $(\log m, \log m+d \log m)$ and $\Gamma$ is the powerlaw "slope" (Bastian et al. 2010). Measurements of the IMF over a wide variety of environments, from young open clusters to field stars, have found the slope to be remarkably consistent with the Salpeter (1955) IMF, $\Gamma_{\text {IMF }}=1.3 \pm 0.2$ for $m>0.5 \mathrm{M}_{\odot}$ (Kroupa 2001). The YMF slope, however, is significantly steeper; $\Gamma_{\mathrm{YMF}}=$ $3.5 \pm 0.6$ above a critical mass, $m \gtrsim M_{c}=3.9 \mathrm{M}_{\odot}$. The YMF apparently flattens to $\Gamma_{\mathrm{IMF}}$ for $3.2 \mathrm{M}_{\odot} \lesssim m \lesssim$ $M_{c}$ before turning over due to incompleteness at lower masses (Fig. 2). Such a steep slope translates into a glaring deficit of massive stars; the YMF contains $>200$ YSOs equivalent to main-sequence B stars $\left(m>3 \mathrm{M}_{\odot}\right)$ but zero $\mathrm{O}$ stars $\left(m>20 \mathrm{M}_{\odot}\right)$, and $\sim 80 \mathrm{OB}$ stars with $m \geq M_{c}$ are missing compared to the predictions of a Salpeter-Kroupa IMF.

\section{DISCUSSION}

\subsection{YSO Mass Spectrum Parameterization}

Assuming a Salpeter-Kroupa IMF applies to M17 SWex, the steep YMF slope can be understood in terms of evolution and selection effects. Our sample is populated by ongoing star formation and includes only YSOs with circumstellar material (disks). We parameterize these effects using the linear form of the mass function, the mass spectrum,

$$
\phi(m)=d N / d m \propto m^{-\alpha}, \quad \alpha=\Gamma+1
$$

(Bastian et al. 2010). The YSO mass spectrum $\psi(m)$ is populated over time as

$$
\psi^{\prime}(m)=\int_{\tau_{0}(m)}^{\tau} \frac{\partial \phi}{\partial t} d t
$$

where $\tau$ is the time since star formation began in the cloud, $\partial \phi(m) / \partial t$ is the star formation rate (SFR) in mass interval $(m, m+d m)$, and $\tau_{0}(m)$ is the time at which

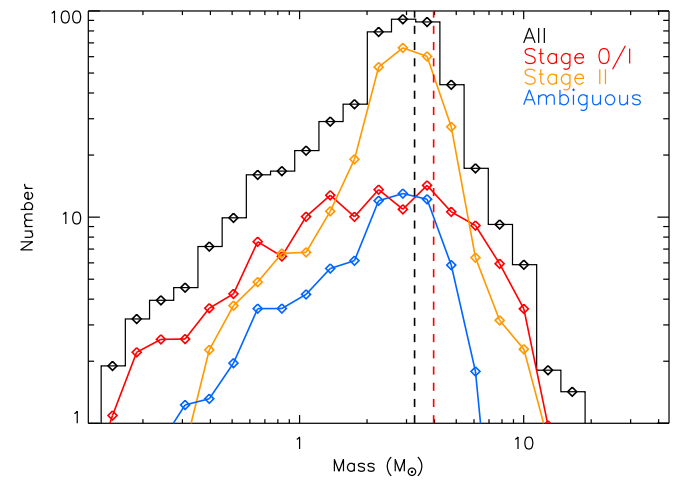

Fig. 3.- YMF of Fig. 2 binned up by a factor of 2 and subdivided by evolutionary stage.

star formation began for each mass interval. Assuming for simplicity that the SFR is not time-dependent, $\partial \phi(m) / \partial t \rightarrow \phi(m) / \tau$ and the integral in Equation 3 becomes

$$
\psi^{\prime}(m)=\phi(m)\left[1-\tau_{0}(m) / \tau\right]=\phi(m) f_{0}(m),
$$

where $f_{0}(m)$ is the fraction of stars that have already formed versus all stars that will eventually form in each mass interval. In the limiting cases of $\tau_{0}(m)=0$ (first stars to form in cloud) or $\tau_{0}(m) \ll \tau$ (timescale for differential onset of star formation small compared to age of population), $f_{0}(m)=1$ and $\psi^{\prime}(m)=\phi(m)$.

The YSO sample is further biased by disk evolution. Taking $\tau_{d}(m)$ to be the disk lifetime as a function of mass,

$$
\psi(m)=\psi^{\prime}(m) \frac{\tau_{d}(m)}{\tau-\tau_{0}(m)}=\psi^{\prime}(m) f_{d}(m),
$$

where $f_{d}(m)$ is the (mass-dependent) disk fraction. For $\tau_{d}(m) \geq \tau-\tau_{0}(m), f_{d}(m)=1$.

Combining Equations 3a and 4

$$
\psi(m)=\phi(m) f_{0}(m) f_{d}(m) .
$$

Since the observed YMF can be fit with a power law (Fig. 2), we parametrize $f_{0}(m)$ and $f_{d}(m)$ as $m^{-\omega}$ and $m^{-\delta}$, respectively. Hence the observed deviation of the YMF slope from the standard IMF is

$$
\Delta \alpha=\Delta \Gamma=\Gamma_{\mathrm{YMF}}-\Gamma_{\mathrm{IMF}}=\omega+\delta=2.2 \pm 0.6
$$

for $m \geq M_{c}$ (Fig. 2).

\subsection{Implications of a Steepened YSO Mass Function: Disk Evolution, Delayed Massive Star Formation, or Both?}

We have shown that a steep YMF could be produced by delayed formation of massive stars or by shorter disk lifetimes for more massive stars. Both interpretations have physical basis. Can either of these effects alone explain the observed YMF?

Disk evolution. The YMF is broken down by evolutionary stage in Fig. 3, and it is apparent that the steep slope is driven by Stage II sources. We thus consider the case of disk evolution alone: $\omega \rightarrow 0, \delta \rightarrow 2.2$ (Equation 6). Hernandez et al. (2007) measured the disk fractions in the $\sigma$ Orionis cluster (age $\sim 3 \mathrm{Myr}$ ) to be $\sim 35 \%$ for $m \leq 1 \mathrm{M}_{\odot}$ and $\sim 10 \%$ for $m>2 \mathrm{M}_{\odot}$, giving $\delta<1.8$. 
This does not rule out disk evolution as the primary driver of the YMF slope, given the uncertainties, but the disk lifetimes themselves, which set the duration of the Stage II phase, become problematically short. Adopting $\delta=1.8$ and $\tau_{d}\left(1 \mathrm{M}_{\odot}\right)=2 \mathrm{Myr}$ (Haisch et al. 2001), we would predict $\tau_{d}\left(4 \mathrm{M}_{\odot}\right)=0.16$ Myr. This is comparable to the typical duration of the envelope-accretion phase in low-mass stars ( 0.1 Myr; Kenyon \& Hartmann 1995). The Stage 0/I objects in our sample span a wide range in mass (Fig. 3), yet the typical accretion timescale is similarly $\tau_{A} \sim 0.1 \operatorname{Myr}\left(\tau_{A}=M_{\star} / \dot{M}_{\text {env }}\right.$, where $\dot{M}_{\text {env }}$ is the accretion rate in the RW06 models; see Table 1 and P09). We expect the maximum age of a $4 \mathrm{M}_{\odot}$ Stage II source in our sample to be $\left(N_{\mathrm{II}} / N_{0 \mathrm{I}}+1\right) \tau_{A} \sim 0.7 \mathrm{Myr}$ $\left(N_{\mathrm{II}} / N_{\mathrm{OI}} \approx 6\right.$ at $m=4 \mathrm{M}_{\odot} ;$ Fig. 3). This longer, more realistic disk lifetime implies $\delta \sim 0.9$.

Delayed Massive Star Formation. Since disk evolution alone cannot explain the observed YMF, delayed massive star formation must also contribute $(\omega \neq 0)$. While diskless low- and intermediate-mass stars are lost among the overwhelming field star population in the IR images, massive stars cannot easily hide within a dense molecular cloud. The Kroupa (2001) IMF, scaled to match the YMF peak (Fig. 2), predicts $>10$ O stars, including at least one early $\mathrm{O}$ star $\left(m>50 \mathrm{M}_{\odot}\right)$; such stars ionize dusty compact and ultracompact $\mathrm{H}$ II regions. The few H II regions in M17 SWex (visible as compact, bright extended sources in Fig. 1) are insufficiently luminous to contain O stars (Elmegreen et al. 1979; Jaffe \& Fazio 1982). We note also that the ratio $N_{\text {II }} / N_{\text {OI }}$ steadily decreases with $m$ for $m \gtrsim 4 \mathrm{M}_{\odot}$ and actually inverts for $m>6 \mathrm{M}_{\odot}$ (Fig. 3), suggesting that the more massive YSOs are preferentially younger. This is not a firm result, however, because it is based on $<20$ sources, and the trend may instead reflect instability of disks around massive YSOs.

While the data presented here do not support more than simple parameterizations, we can constrain the delay timescale for the most massive stars: $\tau_{0}(m>$ $\left.20 \mathrm{M}_{\odot}\right)>\tau$. If the YMF break at $M_{c} \sim 4 \mathrm{M}_{\odot}$ (Fig. 2) is real, not an artifact of incompleteness, then $\psi\left(m \leq M_{c}\right)=\phi\left(m \leq M_{c}\right)$, hence $f_{d}\left(m \leq M_{c}\right)=1$ (Equation 5), and $\tau=\tau_{d}\left(M_{c}\right) \sim 0.7 \operatorname{Myr}(\bar{E} q u a t i o n ~ 4)$ is age of the oldest YSOs in the cloud.

\subsection{Present-Day Star Formation Rate}

Integrating the scaled Kroupa (2001) IMF (Fig. 2) over $m \geq 0.1 \mathrm{M}_{\odot}$ yields a total population of $1.3 \times 10^{4}$ YSOs in M17 SWex, with a total stellar mass of $8 \times 10^{3} \mathrm{M}_{\odot}$. Elmegreen \& Lada (1976) predicted that M17 SWex would eventually form an OB association; these numbers, lower limits due to possible incompleteness, show that the M17 proto-OB association is already forming. Adopting $\tau=0.7 \mathrm{Myr}$, the present-day SFR in M17 SWex is $0.011 \mathrm{M}_{\odot} \mathrm{yr}^{-1}$, comparable to the time-averaged SFR of M17 itself (P09), albeit distributed over a much larger volume.

\subsection{Sequential Star Formation in the M17 Complex}

Elmegreen et al. (1979) compared the morphology of the M17 complex to the dust lanes and "beads-onstrings" H II regions of extragalactic spiral structure. P09 discovered an extended, diffuse H II region northeast of M17, called M17 EB, ionized by a group of optically revealed $\mathrm{O}$ stars in a $2-5 \mathrm{Myr}$ old cluster or association. When M17 SWex is included, the entire M17 complex presents a clear sequence of star formation extending $>100$ pc parallel to the Galactic midplane and spanning several Myr in age. As Elmegreen \& Lada (1976) noted, Galactic spiral density waves propagate in the direction of decreasing age. The difference between the Galactic rotation speed and the spiral pattern speed at the location of M17 is $221 \mathrm{~km} \mathrm{~s}^{-1}-130 \mathrm{~km} \mathrm{~s}^{-1}=91 \mathrm{~km} \mathrm{~s}^{-1}$ (Brand \& Blitz 1993; Martos et al. 2004). The timescale for a spiral shock to cross the entire M17 complex is $\sim 1$ Myr, the same order of magnitude as the observed age spread.

The rapid passage of the M17 complex through the Sagittarius spiral arm served as the "global" trigger ultimately responsible for the formation of this large OB association. Has sequential "local" triggering driven by OB stars (Elmegreen \& Lada 1977) played an important role? While there is strong circumstantial evidence for locally triggered star formation on the periphery of the M17 and M17 EB H II regions (P09 and references therein), it remains unclear whether any of the major OB clusters were similarly triggered. Global triggering appears to be a far more likely explanation for the formation of the proto-OB association in M17 SWex. The $\lesssim 0.7$ Myr spread in ages among the YSO sample is small compared to the 2-3 Myr timescale for sequential triggering by OB stars (Elmegreen \& Lada 1977). The spatial distribution of both the stars and the dust (Fig. 1) suggest a disturbed molecular cloud that is experiencing global collapse and fragmentation.

\section{SUMMARY}

We have catalogued 488 predominantly intermediatemass YSOs in M17 SWex. Analysis of our YSO sample yields the following main results:

- M17 SWex will form >200 B stars. The total stellar population in the cloud is $>1.3 \times 10^{4}$, with total stellar mass $>8 \times 10^{3} \mathrm{M}_{\odot}$. The present-day $\mathrm{SFR}$ is $\sim 0.011 \mathrm{M}_{\odot} \mathrm{yr}^{-1}$. M17 SWex is therefore forming a proto-OB association.

- O stars are conspicuously absent. The YMF slope is significantly steeper than Salpeter for $m>4 \mathrm{M}_{\odot}$.

- Disk evolution proceeds more rapidly for highermass stars, with typical disk lifetimes of $\lesssim 0.6 \mathrm{Myr}$ for YSOs with $m>4 \mathrm{M}_{\odot}$.

- Massive stars begin forming at later times than low-mass stars. M17 SWex probably has not yet formed its most massive star, predicted to be an early O-type star of $m>50 \mathrm{M}_{\odot}$.

If a $>0.5$ Myr delay in the onset of massive star formation is the rule in Galactic giant molecular clouds, the best places to study the initial conditions of massive star formation may be clouds where intermediate-mass star formation has already begun.

We thank T. P. Robitaille for providing the YSO models and SED fitter, M. R. Meade for performing $24 \mu \mathrm{m}$ 
PSF photometry, L. K. Townsley and P. S. Broos for useful conversations, and the anonymous referee for helpful comments. M.S.P. is supported by an NSF Astronomy and Astrophysics Postdoctoral Fellowship under award
AST-0901646. B.A.W. acknowledges support from the Spitzer GLIMPSE programs, Spitzer Theory Program, and NSF AST-0507164.

Facilities: Spitzer, 2MASS, MSX

\section{REFERENCES}

Bastian, N. Covey, K. R., \& Meyer, M. R. 2010, ARA\&A, in press (astro-ph/1001.2965)

Benjamin, R.A. et al. 2003, PASP, 115, 953

Bernasconi, P. A. \& Maeder, A. 1996, A\&A, 307, 829

Beuther, H. \& Sridharan, T. K. 2007, ApJ, 668, 348

Brand, J. \& Blitz, L. 1993, A\&A, 275, 67

Butler, M. J. \& Tan, J. C. 2009, ApJ, 696, 484

Carey, S. J. et al. 2009, PASP, 121, 76

Chini, R. \& Hoffmeister, V. 2008, Handbook of Star Forming Regions, Volume II, B. Reipurth, ed., San Francisco: ASP Monograph Publications p. 625

Cyganowski, C. J. et al. 2008, AJ, 136, 2391

Elmegreen, B. G. \& Lada, C. J. 1976, AJ, 81, 12

Elmegreen, B. G. \& Lada, C. J. 1977, ApJ, 214, 725

Elmegreen, B. G., Lada, C. J., \& Dickinson, D. F. 1979, ApJ, 230,415

Flaherty, K. M. et al. 2007, ApJ, 663, 1069

Haisch, K. E., Lada, E. A., \& Lada, C. J. 2001, AJ, 121, 2065

Hernández, J. et al. 2007, ApJ, 662, 1067

Indebetouw, R. et al. 2005, ApJ, 619, 931

Indebetouw, R. et al. 2007, ApJ, 666, 321

Jaffe, D. T. \& Fazio, G. G. 1982, ApJ, 257, L77

Kang, M., Bieging, J. H., Povich, M. S., \& Lee, Y. 2009, ApJ, 706,83
Kenyon, S. J. \& Hartmann, L. 1995, ApJS, 101, 117

Kroupa, P. 2001, MNRAS, 322, 231

Kurucz, R. 1993, ATLAS9 Stellar Atmosphere Programs and 2

$\mathrm{km} / \mathrm{s}$ grid. Kurucz CD-ROM No. 13. Cambridge, MA:

Smithsonian Astrophysical Observatory

Martos, M., Hernandez, X., Yáñez, M., Moreno, E., \& Pichardo, B. 2004, MNRAS, 350, L47

McClure, M. 2009, ApJ, 693, L81

Price, S. D., Egan, M. P., Carey, S. J., Mizuno, D. R., and Kuchar, T. A. 2001, AJ, 121, 2842

Povich, M. S. et al. 2009, ApJ, 696, 1278 (P09)

Rathborne, J. M., Jackson, J. M., \& Simon, R. 2006, ApJ, 641, 389

Robitaille, T. P., Whitney, B. A., Indebetouw, R., Wood, K., \& Denzmore, P. 2006, ApJS, 167, 256 (RW06)

Robitaille, T. P., Whitney, B. A., Indebetouw, R., \& Wood, K. 2007, ApJS, 169, 328

Salpeter, E. E. 1955, ApJ, 121, 161

Smith, N. et al. 2010, MNRAS, in press

Siess, L., Dufour, E., \& Forestini, M. 2000, A\&A, 358, 593

Whitney, B. A. et al. 2008, AJ, 136, 18 